\title{
Smart meters as a key component of modern measuring infrastructure providing observability and state estimation of low-voltage distribution networks
}

\author{
Yana Kuzkina $^{1^{*}}$, and Irina Golub ${ }^{1}$ \\ ${ }^{1}$ Melentiev Energy Systems Institute of Siberian Branch of Russian Academy of Sciences, Irkutsk, Russia
}

\begin{abstract}
The paper presents a solution to the problem of organization of a system for collecting and transmitting information about measurements from smart meters necessary for the state estimation of a lowvoltage distribution network. The problems of providing the sufficiency of measurements for the observability of the network and the influence of errors in the information about load connection to phases on the quality of the observability are considered. The results of allocation of smart meters and the state estimation of the real distribution network are given.
\end{abstract}

\section{Introduction}

Efficiency and reliability of energy use can be ensured by using information about the current load condition coming from the measuring infrastructure to the control centre of the electric power system (EPS) in real time. Measurement data are processed by state estimation (SE) algorithms that allow to refine the measurements and to calculate all the variables of the EPS state required for monitoring and control. A necessary condition for ensuring the existence of a solution to the problem of SE according to measurements is the EPS observability. The quality of observability, which determines the influence of measurement errors on the solution obtained during SE, depends on a set and an allocation of measurements. It can be improved by increasing the number of measurements.

Methods of SE have found a wide application in high and ultra-high voltage networks, through which electricity is transferred from power plants to distribution networks (DNs) directly connected with consumers. For the SE in such networks Phasor Measurement Units (PMUs) are used allowing simultaneous and synchronized vector measurements in remote nodes using GPS or GLONASS. PMU measures the voltage vector in the node and the current vectors in adjacent branches [1]. The results of the SE based on PMU can provide information about the power entering the primary medium-voltage (MV) DN. Neither total loads of the transformers nor, especially, the loads of the secondary low-voltage (LV) DN can be obtained.
2 Preparation of information for the state estimation of the secondary distribution network

In the traditional DN, only the measurements at primary distribution substations on the MV side are available to the dispatcher, as well as measurements of critical loads [3]. Obviously, such measurements are not enough either for the SE of DNs or for the calculation of the load flow. Passive DNs gradually become active ones with a difficultly predictable state owing to introduction of renewable generation sources, energy storage and active consumers controlling their consumption depending on tariffs. There is a need to equip DNs with measuring devices and conduct the SE of DN on their basis. The SE of the primary DN is necessary for solving such problems as estimation of energy losses, reactive power and voltage regulation, network reconfiguration and load forecasting. In the secondary DN information about load condition is used for voltage and power flow control, also for calculations of power losses, short-circuit currents and reliability indicators [2]. The SE of the secondary DN can be the basis for determining voltages and powers entering the secondary $\mathrm{DN}$ in the nodes of connection of transformers to the primary DN. It is actually equivalent nodal powers of the primary DN. Such information will also be the basis for the SE of the primary DN that has no measuring devices. 


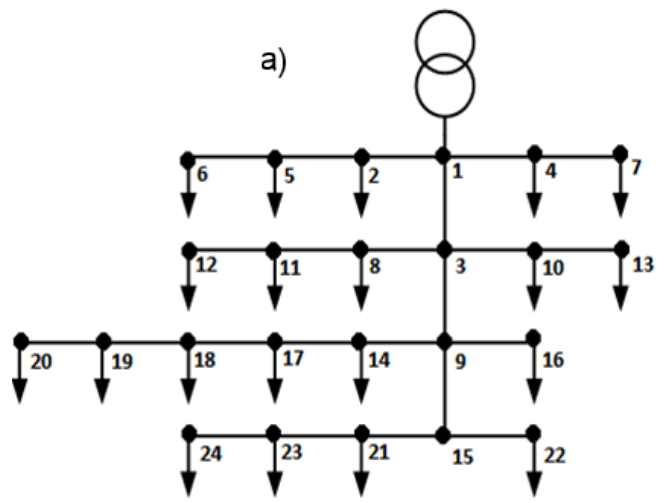

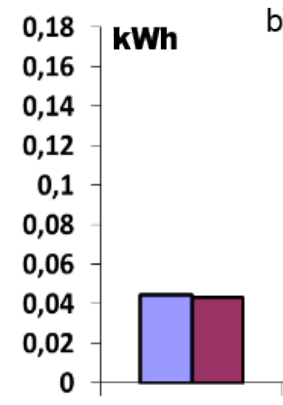

b)

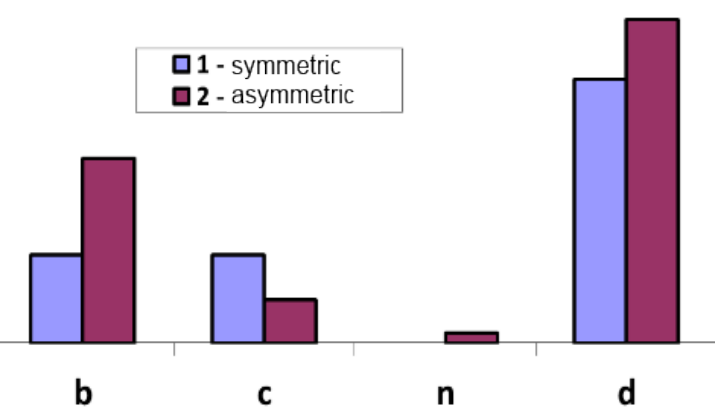

Fig. 1. A scheme of a 24-node feeder of the low-voltage distribution network (a) and a diagram of the results of calculating the hourly energy losses in the feeder (b) for the modes of symmetrical (1) and asymmetrical (2) loads.

Nowadays there is an automated commercial electricity metering system in secondary DNs, the main component of which are electronic meters that measure energy consumption for a certain period of time (a day, a week, a month, a season, a year). Owing that, there is an opportunity of using the information about average loads to calculate the load flow in the DN and to determine the energy losses. [4]

Since there is no information in reports of automated commercial electricity metering systems about loads' distribution by phases, the energy losses estimation will depend on the way the loads are distributed between phases. It is shown with calculations of the three-phase load flow for a 24-node low-voltage feeder of a real DN, Fig.1a.

Two variants of specifying feeder loads were investigated. In the first symmetrical variant nodal loads were assumed to be three-phase symmetrical: the load of the node, single-phase or three-phase, was divided equally between phases. When calculating the load flow in this case, the current in neutral wire and the energy loss in it are zero. In the second asymmetrical load variant, three-phase nodal loads were divided equally between phases and each next single-phase nodal load was assigned to a new phase of the feeder. Feeder loads with this simulation become unbalanced and when calculating the load flow, currents and energy losses appear not only in the neutral wire, but also in the earth. The results of calculating the hourly energy losses in phases $a, b, c$, in the neutral wire $n$ and total losses $d$ for the symmetrical (1) variant and asymmetrical (2) one are shown in the diagram, Fig.1b. Total energy losses in phases for a symmetrical load equal to $0.1336 \mathrm{kWh}$, it is less than the losses for an asymmetrical load - 0.1639
$\mathrm{kWh}$, which also includes energy losses in the neutral wire and in the ground.

It will be shown below that the problem of phase identification also arises when measurements from SMs are used for the SE. To determine the phase, a method is proposed in [5] consisting of a synchronized measurement over a long period of time of the voltage magnitudes in the nearest to the power source control node with known phases and in the SM connection node. The phase is determined from the maximum values of cross-correlation coefficients between two vectors containing measured voltage magnitudes.

The effectiveness of the proposed approach can be illustrated for the fragment of the DN [6], presented in Fig.2a. The node 2, which is the nearest to the power supply node, is taken as a control node with known phases. Nodes 2,9,10,11 have three-phase loads; node 16 in phase $B$ and node 24 in phase $C$ are generator; nodes 5,29,17,25 have single-phase loads, and node 18 - twophase load. For all the nodes, according to the test load measurements for each hour of the day, the information about voltage magnitude was obtained using the threephase SE program.

Further, for the voltage vectors of phases $A, B, C$ of the node 2 and each of the measured vectors of voltages for example, in node 5, the standard MATLAB function corrcoef was used for calculation of cross-correlation coefficient $A A, B A, C A$. The coefficients recorded in table, Fig.2b, suggest that the SM is set in phase $A$.

The phase identification for SM can be determined from the maximum values of correlation coefficients. For example, comparing the correlation coefficients $A A$ (0.9984), $A B$ (0.7757), and $A C(0.9115)$ for the node 5 allows affirming meter is in phase $A$.

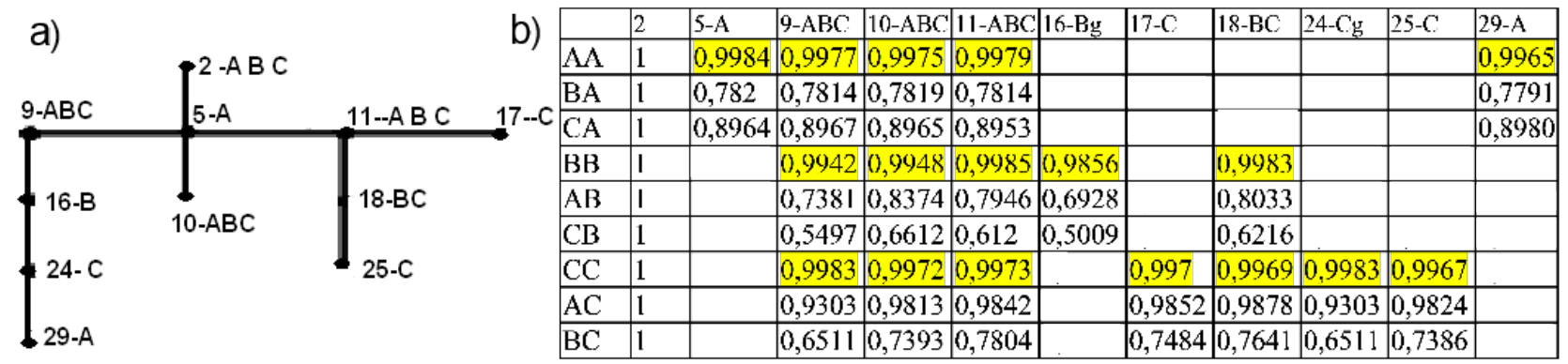

Fig. 2. A fragment of the feeder of the low-voltage distribution network with the phases of connection of loads and generations (a) and the table of cross-correlation coefficients (b). 


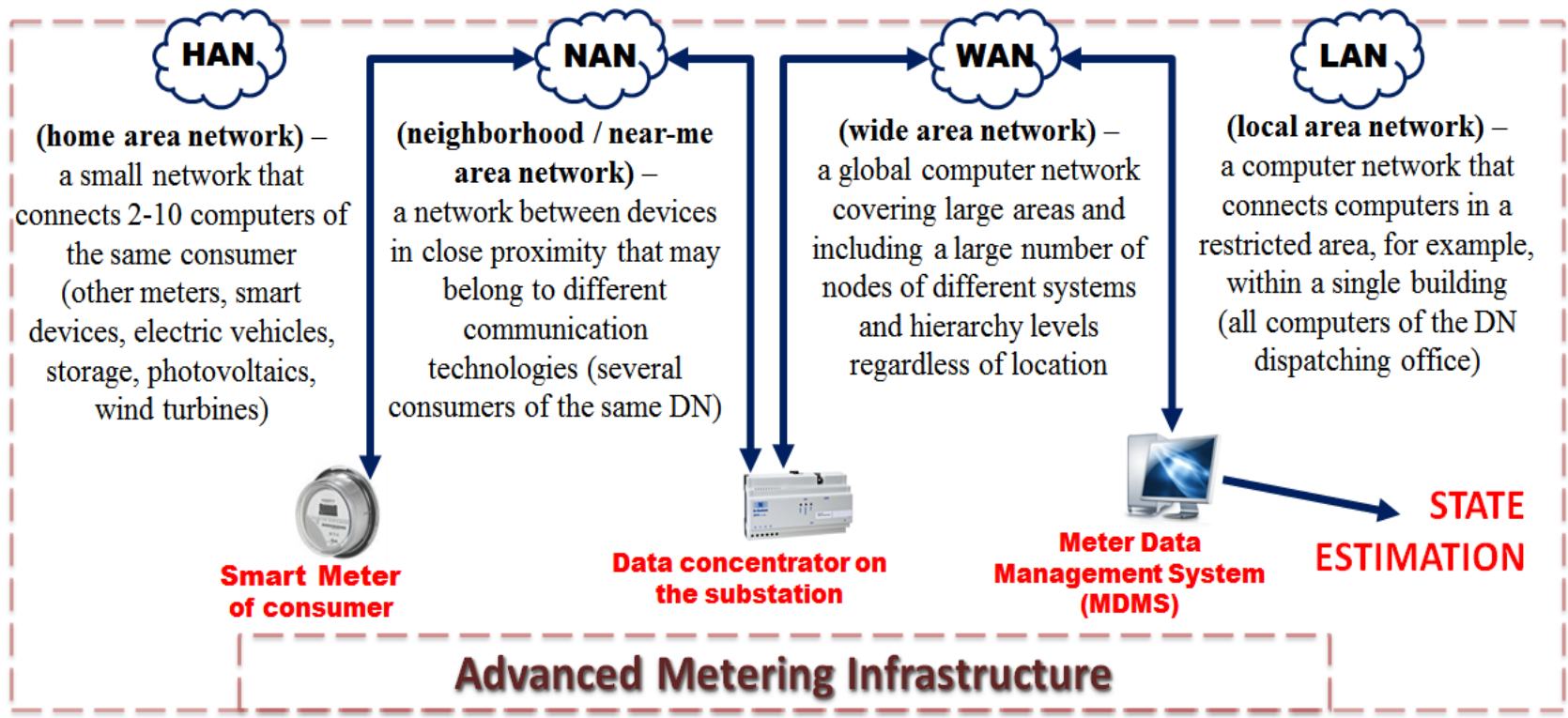

Fig. 3. AMI hierarchy for collecting, transmitting and processing measurements from smart meters.

Additional problems associated with the automated commercial electricity metering system are non-synchronization of measurements, erroneous data about network topology, erroneous data about connection of feeders to secondary transformers. The use of such information is problematic for the calculation of the load flow, and even more so for the SE.

The transition to the concept of smart grid including the use of new electronic devices, such as SMs, and the organization of a universal communication infrastructure for the collection and processing of measurement information, allows the organization of the functioning of the DN in a dialogue format. The control object (a DN dispatcher) does not just send one-way commands, but receives from the control object a message about the possibility of their implementation. As a result, the command can be corrected or cancelled. That will avoid losses and ensure the reliability of the DN operation [7]. To realize the two-way interaction, SMs have been developed, their measurements will form the basis of the three-phase SE of the secondary DN, and, as noted above, the SE of the primary DN.

\section{Smart meters and Advanced Metering Infrastructure}

The appearance of three-phase and single-phase SMs installed in the load and/or generator nodes of LV DN allows obtaining the measurements necessary for the calculation of the load flow and the SE [8]. The transmission and processing of measurements from SMs is possible thanks to the innovative communication system containing various communication technologies Advanced Metering Infrastructure (AMI). AMI collects and analyzes data from SMs, providing two-way communication with a dispatching office, as well as between consumers and utility services. The appearance of AMI and its earlier modifications is the first step towards the transition to digital control systems for DNs. $[8,9]$ Various communication tools are used for AMI organization [9]. In Russia and Europe, the most widely spread wire technology is PLC, associated with the transmission of measured information through the power lines [10], its drawbacks include a bandwidth limitation and long-term responses to incoming messages from SMs. The most common wireless communications are ZigBee [11] and cloud technologies [12]. In Kazakhstan and Belarus, cellular is used for communication between SMs [13]. Wireless technologies are considered to be less expensive, but the range and correctness of signal transmission is limited, the data transfer rate is low, and the penetration and avoidance capacity of the obstacles is weak. In addition, wireless technologies can interfere with other devices. Typically, the PLC is used to transfer measurements to data concentrators located in primary substations. Communication between meters of a single feeder or a section of the DN is carried out via wireless channels. AMI is constructed hierarchically in accordance with the information levels of the computer network (HAN, NAN, WAN, LAN), Fig.3 [7.8].

The review of papers on problems of AMI organization, allocation of SMs in the LV DN and their use for the SE $[2,14]$, allowed to establish the following facts. At the initial stage of the study [15] of the SE problem, measurements from SMs were considered as synchronized vector measurements of nodal voltages and current injections $[16,17]$. It was believed that to determine the location of a particular SM it is possible to use the information attached to it, including the name of the substation, the node number, feeder and phase numbers [18]. However, more recent publications $[7,9,19,20]$ present conflicting information on the problem of synchronization of measurements from SMs and the absence of a specialized standard that determines the sampling rate for SMs. Thus, measurements from SMs are non-synchronized. These are the same electronic meters that are connected to the automated commercial electricity metering systems, performing an average measurement of energy consumption for a certain time interval (15, 30, $60 \mathrm{~min})$. In addition, SMs of several manufacturers can measure the voltage 
magnitude and active and reactive power injections and/or active and reactive current injections in the node with SM. Also it can measure the output power delivered to the supply network in DNs with renewable generation sources.

\section{Allocation of measurements to provide observability and state estimation in distribution networks}

To arrange the measurements by the criterion of topological observability for each phase of LV DN, it is sufficient to know the phase topology and the nodes which are load and/or generator ones. Transit nodes, which are adjacent to two branches, and hanging nodes without nodal power are not included to the equivalent circuit of the phase.

The set of SMs including voltage magnitude and current injection measurements in the node can be determined with the developed program for allocation measurements that ensure the observability of DNs. The algorithms used in the program [21] are based on the algorithms for choosing the set and locations of PMU measurements proposed in [22] to ensure observability in various cases for load condition. In contrast to PMUs, SMs measure not vectors, but magnitudes of voltage. SMs must be installed in all load and/or generator nodes to ensure the observability of the LV DN [6]. Measurements are not installed in transit nodes. If it is not possible to allocate measurements at all load and/or generator nodes, a minimal set of SMs can be used to control nodal voltages. That is especially important in networks with renewable generation. The minimal set of SMs can be obtained using the algorithm for selecting the minimum set of measurements from single-channelled PMUs as shown in [21]. Additional constraints were introduced in the algorithm to prohibit installation of measuring devices in transit nodes adjacent to more than two branches and installation more than one measuring device in the node. After checking

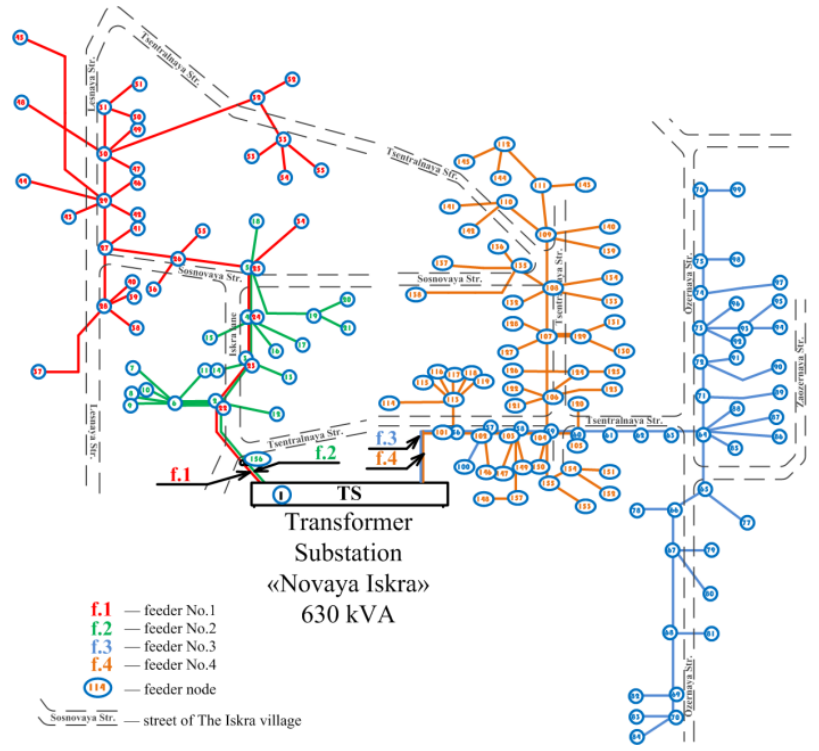

Fig. 4. Fragment of $0.4 \mathrm{kV}$ distribution network of the Iskra village, Irkutsk, Russia. the choosing set of SMs in the topological observability analysis program, such measurements can be used for the SE of each phase of the LV DN. To ensure the observability of the DN with the minimum set of SMs direct-axis voltage components equal to measured voltage magnitudes can be specified as the measurements from SMs. Quadrature-axis voltage components are assumed to be zero. Such replacement is allowable as quadrature-axis voltage components are almost equal to zero. That makes it possible to provide observability, specifically voltage control of only directaxis voltage components in all nodes of the $\mathrm{DN}$, as shown in [15]. A similar replacement is also used when SMs is allocated in all load and/or generator nodes.

A linear SE algorithm based on the extended Hatchtel matrix can be used for the SE of each phase in three-phase four-wire DNs with asymmetrical phase loads, as shown in [23]. The advantage of this method is the elimination of measurements of zero current injections from the objective function and the use of an additional constraint on zero equalization of the weighted residues.

\section{The illustration of the algorithms for choosing the set of measurements and state estimation of the secondary distribution network}

A real LV DN $0.4 \mathrm{kV}$, which belongs to a regional state unitary power company "Oblkommunenergo" (Irkutsk, Russia) is used to illustrate the developed algorithms. It feeds consumers of the Iskra village. The power supply to the Iskra village is provided by four transformer substations, which are supplied from the primary substation, $110 / 6 \mathrm{kV}$, owned by "Irkutsk Electric Grid Company".

Four $0.4 \mathrm{kV}$ feeders of substation "Iskra-Novaya" were taken for the calculations, Fig.4: feeder No.1 - 34 nodes, feeder No. 2 - 21 nodes, feeder No. 3 - 45 nodes, feeder No.4 - 56 nodes. The scheme includes 157 nodes taking into account the power node, 60 nodes are transit. "Oblkommunenergo" gave necessary information for the study about the network topology, brands of electric wires and line lengths; a list of consumers.

During the preparation of the scheme for calculating the state, problems were found that are typical for the most real DNs in Russia. The geographical maps (2GIS, Yandex and Google) available on the Internet contain conflicting information about house numbering and street names, and in the given list of consumers not all addresses are presented in an understandable form and correspond to the map data. Despite the existence of the list of consumers, there is no information on their belonging to a particular phase. When analyzing and processing the provided data on energy consumption for various periods of time, it was found that consumption readings are either mis-transmitted by users or contain errors that occur when recording information, which can even lead to negative consumption.

Since the exact consumption is unknown, two versions of the initial data were generated for the state 


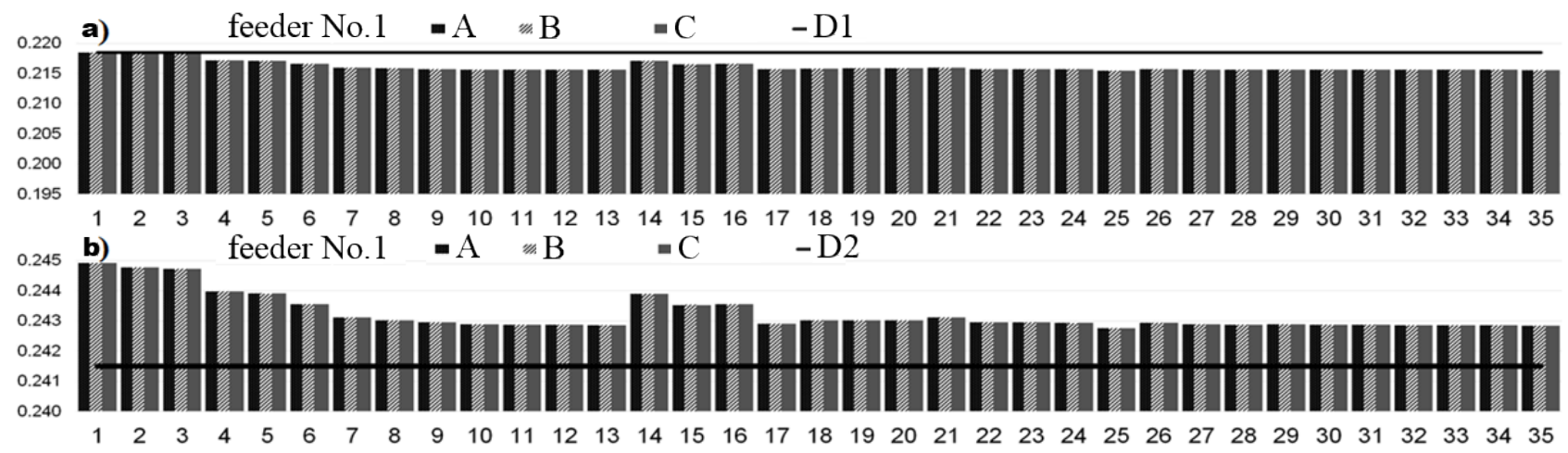

Fig. 5. Comparison of the test state data and estimates for two sets of SMs in the feeder No.1 of the $0.4 \mathrm{kV}$ DN in the Iskra village for initial data No.1 (a) and No.2 (b); values of direct-axis voltage components for: A - test mode (steady-state operational mode); B - the minimum set of SMs; C - SMs in all load nodes; D1 and D2 - minimum and maximum allowable voltages.

analysis. In variant No.1, the loads were set in accordance with the available limit in Russia for the power consumption for domestic needs of $15 \mathrm{~kW}$. This load was accepted for three-phase consumers. Singlephase loads were taken equal to $5 \mathrm{~kW}$. The reactive component of the nodal power was adopted on the basis of the reactive power factor normalized for utility networks $(\operatorname{tg} \varphi=0.2)$. The voltage on the low side of the transformer substation was set at $0.38 \mathrm{kV}$. A single-phase state for the DN was calculated, which showed that, for example, in feeder No.1 in 32 nodes voltage deviation exceeds $10 \%$, which is confirmed by the estimates of "Oblkommunenergo" employees. At the moment, the company has already developed a technical task for the network reconstruction of the Iskra village and the development of the project has begun, including the addition of a substation and the reconfiguration of the entire network. In the version of state No.2 to simulate the possible overvoltages in the network, which can be further determined by the SE, the values of the node load powers were reduced by $20 \%$, the voltage on the low side of the transformer substation was taken at $0.425 \mathrm{kV}$, and for a number of the most remote feeder nodes reactive power sources were installed.

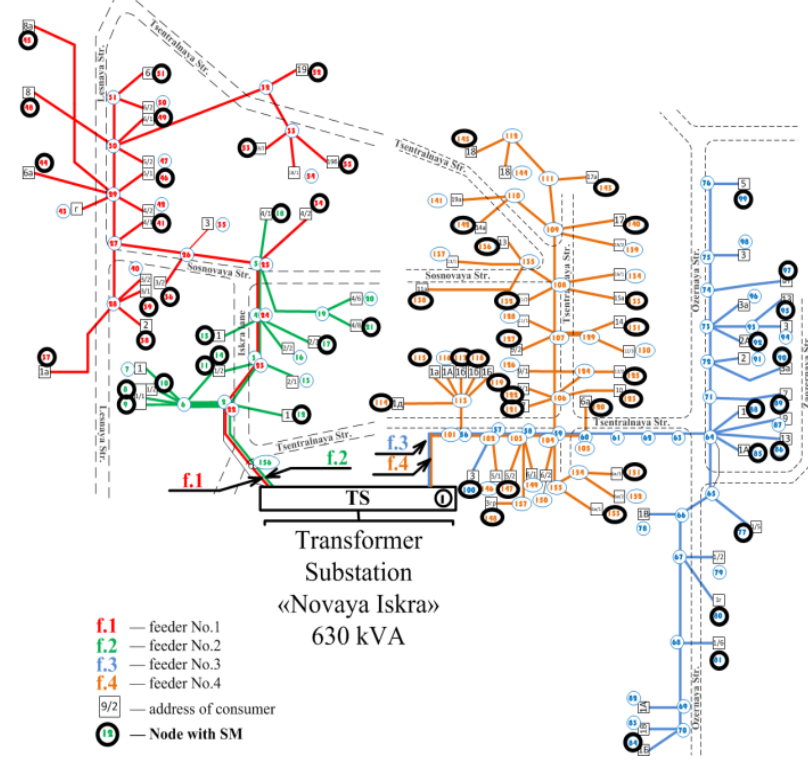

Fig. 6. The minimum set of SMs for the four feeders of the distribution network of the Iskra village.
The allocation of SMs according to the program [21] was made for each feeder independently. When determining the minimum set of the measurements, it becomes possible to edit the allocation of the SMs selected by the program: for example, to replace the meter installation in the garage allocated in the scheme as a load node (node 43, Fig.4), to install the SM in the residential building (node 44, Fig.4). For the DN, Fig.4, the minimum measurement set is obtained, including 64 SMs, and the SM setting is determined in all 96 load nodes.

As measurements for the SE, injections of active and reactive currents and voltage magnitudes were used, while errors of $0.4 \mathrm{~V}$ and $0.3 \mathrm{~A}$ were introduced into the test values. Fig. 5 compares the direct-axis components of the nodal voltages of the test state of the feeder No.1 with estimates obtained from the linear SE program for two sets of SMs for the initial data No.1 (Fig.5a) and No.2 (Fig.5b).

In the first case, in all nodes of the feeder there are unacceptable voltage drops, in the second case overvoltages. The analysis of the SE results confirmed the conclusion obtained earlier that even the minimum set of SMs makes it possible to obtain estimates of nodal voltages with acceptable accuracy and to reveal their deviations. The maximum error of estimates in comparison with the test state data was $0.14593 \%$ for the case No.1 and $0.09117 \%$ for the case No. 2.

At this stage of the study a minimum number of SMs can be proposed for four feeders of the LV DN departing from "Iskra-Novaya" substation of "Oblkommunenergo". Such set, including 64 SMs, installed in the circled nodes, Fig.6, is sufficient to control voltage levels in the LV DN of the Iskra village.

\section{Conclusion}

The problems that arise while prepare the information for SE of the secondary DN are analysed, such as:

1. the choice of measuring devices and the associated set of measured variables;

2. the identification of phases of consumers' connection;

3. the ability to synchronize measurements;

4. errors in information about the topology of the DN and the connection of feeders to transformers. 
Methods for constructing a modern measurement infrastructure including an active $\mathrm{DN}$, a communication network and measurements of smart meters that are used not only for collecting information about energy consumption but also for the SE in a secondary DN are considered.

The problem of providing the observability of all variables of the secondary DN based on SMs and the observability of only the magnitudes of node voltages is considered.

The proposed approaches were applied for choosing the set of SMs and for the SE of the DN, which is managed by the regional state unitary power company "Oblkommunenergo", Irkutsk, Russia.

Recommendations for the allocation of measurements and the organization of a system for the collection and transmission of measured data for the planning and further development of this network can be given to "Oblkommunenergo" based on the results of the study. The most important moments are the need to install smart meters in all load nodes, to ensure the synchronization of such measurements and the required knowledge of the phase connection of consumers.

The work is done in the framework of the project III.17.4.2. program of fundamental research SB RAS, registration number AAAA-A17-117030310438-1.

\section{References}

1.R.F. Nuqui, A.G Phadke, IEEE Power Tech., 4 (2007)

2.A. Primadianto, Ch.-N Lu, IEEE Trans. Power Syst., 32, 5 (2017)

3.F. Ni, P.H. Nguyen, J.F.G. Cobben, H.E. Van den Brom, D. Zhao, INT J ELEC POWER, 98, 463-473 (2018)

4.Ismerenie.RU, 16 (2013) [online] URL: https://www.izmerenie.ru/ru/number-15-6-12 (accessed: 30.06.2018)

5.H. Pezeshki, P.J. Wolfs, ISGT Europe, 3, 1-7 (2012)

6.P.C. Olival, A.G. Madureira, M. Matos, EPSR, 146, 132-140 (2017)

7.S. Ma, H. Zhang, X. Xing, Wireless Personal Communications, 99, 1 (2017)

8.Le T.N., Chin W-L., Truong D.K., Nguyen T.H. , Smart Metering Technology and Services, Chapter 3, (IntechOpen, 2016)

9.R.R. Mohassel, A. Fung, F. Mohammadi, K. Raahemifar, CCECE, 8 (2014)

10.A. Sendin, I. Berganza, A. Arzuaga, X. Osorio, I. Urrutia, P. Angueira, Energies, 6 (2013)

11.S.-W. Luan, J.-H. Teng, S.-Y. Chan, L.-C. Hwang, PEDS, 661-665 (2009)

12.K. Billewicz, MEPS, 1-6 (2015)

13.Ismerenie.RU, 17 (2013) [online] URL: https://www.izmerenie.ru/ru/number-15-6-12 (accessed: 30.06.2018)
14.A.A.M. Raposo, A.B. Rodrigues, M. Silva, EPSR, 147 (2017)

15.I.I. Golub, Y.I. Kuzkina, Mathematical models and methods of the analysis and optimal synthesis of the developing pipeline and hydraulic systems, 15, 182192 (2016)

16.S.S.S.R. Depuru, L. Wang, V. Devabhaktuni, IEEE/PES Power Systems Conference and Exposition, 15, 2736-2742 (2011)

17.S. Santos, A Llano, A. Arzuaga, T.Arzuaga, L.Marron, M.Zamalloa, CIGRE, 8 (2012)

18.Sioe T. Mak. Power and Energy Society General Meeting, 1-3 (2011)

19.S. Rohrenbeck, Construction, Commissioning and Use of a Test Bench for Smart Meter Accuracy Verification (IEEE PESS, Dortmund, 2015)

20.E. Bompard, A. Bahmanyar, E. Patti, ICEE, 24, 10821087 (2016)

21.I.I. Golub, Y.I. Kuzkina, Acta Energetica, 4/33, 4-9 (2017)

22.I.I. Golub, M.V. Khokhlov. Elektrichestvo 1, 8 (2015)

23.L. Holten, A. Gjelsvik, S. Aam, F.F.Wu, W.-H.E. Liu, IEEE Trans. Power Syst., 3, 4 (1988)

24.E.V. Boloev, I.I. Golub, V.V. Fedchishin, PROCEEDINGS of ISTU, 22, 2 (2018) 